\title{
FRACTIONAL COSINE TRANSFORM (FRCT)-TURBO BASED OFDM FOR UNDERWATER ACOUSTIC COMMUNICATION
}

\author{
Yixin Chen \\ Centre for Excellence in Signal and Image Processing (CeSIP) \\ University of Strathclyde \\ Glasgow, UK \\ yixin.chen@strath.ac.uk \\ John. J. Soraghan \\ Centre for Excellence in Signal and Image Processing (CeSIP) \\ University of Strathclyde \\ Glasgow, UK \\ j.soraghan@strath.ac.uk
}

\author{
Carmine Clamente \\ Centre for Excellence in Signal and Image Processing (CeSIP) \\ University of Strathclyde \\ Glasgow, UK \\ carmine.clemente@strath.ac.uk \\ Stephan Weiss \\ Centre for Excellence in Signal and Image Processing (CeSIP) \\ University of Strathclyde \\ Glasgow, UK \\ stephan.weiss@strath.ac.uk
}

\begin{abstract}
In this paper, a hybrid Discrete Fractional Cosine Transform (DFrCT) with Tikhonov regularization based Turbo Minimum mean square error (MMSE) equalization (DFrCTTurbo) is presented to suppress inter-carrier interference (ICI) over underwater acoustic channels (UWA). The scheme is based on Orthogonal Frequency Division Multiplex (OFDM) scenario. In addition, an optimal order selecting method for DFrCT is developed by maximizing carrier to interference ratio (CIR) to UWA channel character. Simulation results show that BER improvement of up to $5 \mathrm{dBs}$ over traditional orthogonal based methods with moderate complexity.
\end{abstract}

Keywords-Discrete Fractional Cosine Transform (DFrCT), carrier to interference ratio (CIR), Tikhonov regularization based Turbo Minimum mean square error (MMSE) equalization

\section{INTRODUCTION}

There are two main obstacles of communication over underwater acoustic channel (UWA)[1], significant time delays that may reach fractions of seconds and severe Doppler spread mobile attributed to high mobile between transmitter and receiver. An increase in the number of subcarriers in OFDM systems contributes to a decrease of subcarrier spacing bandwidth. Consequently such schemes are subject to suffer from loss of orthogonality of subcarriers, caused by highly Doppler spread, contributing to Inter-Carrier-Interference (ICI)[2]. Research has focused on the suppression of ICI with low complexity, which can be divided into prior FFT and post FFT methods respectively [2]. Post FFT mainly deal with equalization techniques. By exploring the band nature of frequency channel matrix, block band MMSE (BMMSE) are used[3], in which symbols are jointly equalized in a block, and its complexity is linear to block length, superior to that of conventional MMSE, growing cubically in subcarrier numbers.

Based on BMMSE, the performance could be further improved in combination with interference cancellation, such as maximum-likelihood and decision feedback approaches.

Iterative detection and decoding techniques were introduced in [4]-[6], such as the block turbo equalizer based on the exchange of soft extrinsic information [most often loglikelihood ratios (LLRs)] between MMSE equalization, and maximum a posteriori probability (MAP) decoder. Banded equalization with iterative data detection has a superior robustness against errors of channel estimation, which usually occurs in the UWA scenario [7]-[8].

To compete with the doubly selective (both time and frequency selective) nature of UWA channels, in our previous work [2] the conventional FFT demodulation in the OFDM was replaced by the Fractional Fourier Transform (FrFT) [9] which transforms the signal into an intermediate domain between time and frequency. Doubly selective channel response were shown to be concentrated into narrower bands, allowing ICI in adjacent subcarriers to be concentrated around main diagonal of channel frequency matrix.The Fractional Cosine Transform (FrCT), based on the eigen-decomposition of the DCT kernel, shows a better energy concentration than FrCT and FFT with less complexity [10].

In this paper, a novel block turbo MMSE equalization method for OFDM scenario based on Discrete Fractional Cosine Transform (DFrCT), called DFrCT-Turbo is presented. An optimal order selecting scheme for DFrCT is provided. At the analysis stage, a doubly selective channel scenario is simulated and the results show the superior performance of the DFrCT-Turbo approach compared to conventional turbo equalization and DFrFT-OFDM. Moreover, the BER 
performance is further improved by incorporating convolutional error correction coding.

The remainder of paper is organized as follows. Section 2 presents the proposed DFrCT-Turbo based algorithm, with a DFrCT-OFDM block, a low complexity block turbo equalization based on Tikhonov regularization, and an optimal order selecting scheme. In section 3, simulation results and discussions are reported. Section 4 gives a conclusion.

Notation: In this paper, transpose, conjugate and conjugate transpose are denoted as $[.]^{T},[.]^{*}$ and $[.]^{\mathrm{H}}$ respectively. $\operatorname{diag}\{$.$\} denotes a diagonal matrix produced by a vector$ $[.]_{i, j}$ extracts the $i$ th row and $j$ th column element from a matrix. Finally, $\quad\langle.\rangle_{N}$ is the modulo-N calculation. ||. ||indicates the Frobenius norm

\section{System model}

\section{A. DFrCT-Turbo Transceiver}

The proposed DFrCT-Turbo system shown in Figure 1 consists of several blocks. At first, switch 1 is open and the system works in optimal order selection model to search the optimal order $\boldsymbol{\alpha}_{\mathbf{o p t}}$ range from -1 to 1 . An initial order of -1 is used. The input signal $s_{n}$ is passed through conventional rate $1 / 2$ ECC and interleaver modules. The encoded data vector is then transformed into time domain using an inverse DFrCT of order $\alpha$. The separate parallel data streams are then converted to a serial data after adding cyclic prefix. The serial received data stream is corrupted by a doubly selective channel with additive white Gaussian noise n. The output of the channel is fed into the DFrCT optimal order selection module. The optimal order $\boldsymbol{\alpha}_{\text {opt }}$ is feedback to transmitter. Then, the switch 1 close and the system works on equalization model, the received signal at optimal order $\boldsymbol{\alpha}_{\text {opt }}$ is converted to a parallel data stream, which is processed by the DFrCT after discarding cyclic prefix. Then, the Tikhonov regularization based turbo MMSE equalization is carried out on each block of symbols jointly, and iteratively interchanges extrinsic information with the convolutional decoder.

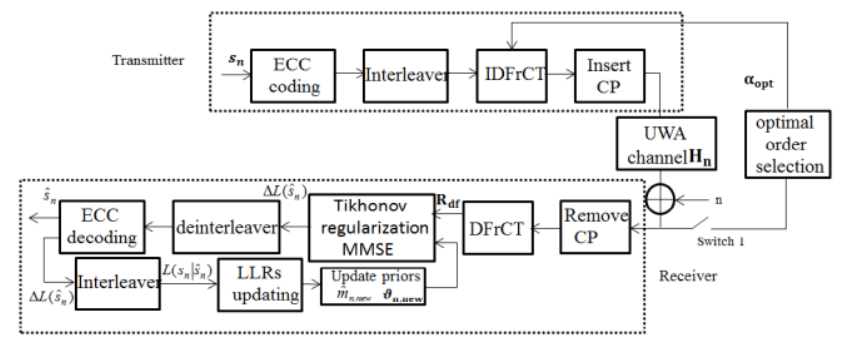

Figure1: Diagram of DFrCT-Turbo Transceiver

\section{B. Discrete Fractional Cosine Transform (DFrCT)}

The Discrete Fractional Cosine Transform (DFrCT) [11], a generalization of the cosine transform (DCT), transformed a function into an intermediated domain between time and frequency, according to the rotation of time-frequency distribution via angle or order. In our previous work [10], the
DFrCT of order is expressed in matrix vector notation as below:

$$
y_{\alpha}=F_{\alpha} x
$$

Where

$$
\begin{gathered}
\boldsymbol{y}_{\boldsymbol{\alpha}}=\left[y_{\alpha}[0], y_{\alpha}[1] \ldots y_{\alpha}[N-1]\right]^{T}, \\
\boldsymbol{x}=[x[0], x[1] \ldots x[N-1]]^{T}
\end{gathered}
$$

and $\mathbf{F}_{\boldsymbol{\alpha}}$ is the $\mathrm{N}$ point Fractional Cosine Transform matrix. Similarly, the inverse DFrFT is written as

$$
\boldsymbol{x}=\boldsymbol{F}_{\alpha}^{\boldsymbol{H}} \boldsymbol{y}_{\alpha}=\boldsymbol{F}_{-\alpha} \boldsymbol{y}_{\alpha}
$$

It represents one of the main components of the proposed algorithm and is described in the next section.

\section{Discrete Fractional Cosine Transform (DFrCT) OFDM system}

The DFrCT based OFDM system, replaces both the Discrete Fourier (DFT) and inverse Discrete Fourier Transform (IDFT) blocks by the DFrCT and IDFrCT respectively. The $\mathbf{N}_{\mathbf{a}}$-block binary sequence mapped by BPSK constellation $\boldsymbol{s}=\left[s_{1} \ldots s_{N_{a}}\right], s_{n} \in{ }_{-}^{+} 1$, is allocated into $\mathrm{N}$ subcarriers via a binary matrix, denoted as

$$
\boldsymbol{J}=\left[0_{N_{a} X\left(N-N_{a}\right) / 2} \boldsymbol{I}_{\boldsymbol{N}_{\boldsymbol{a}}} 0_{N_{a X(N-N a) / 2}}\right]^{T}
$$

where $\mathbf{0}_{\mathbf{L x M}}$ is an LxM diagonal zero matrix, and $\mathbf{I}_{\mathbf{L}}$ is an $\mathrm{LxL}$ identity matrix.

Then, the signal, modulated by the IDFrCT, is corrupted by a doubly selective (DS) channel. After removing cyclic prefix, the received signal $\mathbf{R}_{\mathbf{n}}$ is expressed as

$$
\begin{aligned}
& R_{n}=H_{n} s_{n}+v_{n} \\
& \quad=H_{n} F_{-\alpha} J d_{n}+F_{\alpha} v_{n}
\end{aligned}
$$

where $\boldsymbol{H}_{n}$ represents the time domain channel matrix characterized by

$$
\left[\boldsymbol{H}_{\boldsymbol{n}}\right]_{i, j}= \begin{cases}h[n-L+i, i-j] & i \geq j, \\ h[n-L+i, L+i-j-1] & i<j\end{cases}
$$

The received signal after DFrFT demodulation can be then expressed as

$$
\begin{aligned}
\boldsymbol{R}_{d f} & =J^{H} F_{\alpha} H_{n} s_{n}+F_{\alpha} v_{n} \\
& =J^{H} F_{\alpha} H_{n} F_{-\alpha} J d_{n}+F_{\alpha} v_{n} \\
& =J^{H} H_{d f} J d_{n}+F_{\alpha} v_{n}
\end{aligned}
$$

According to the frequency channel matrix $\boldsymbol{H}_{\boldsymbol{d f}}=$ $\boldsymbol{F}_{-\boldsymbol{\alpha}} \boldsymbol{H}_{\boldsymbol{n}} \boldsymbol{F}_{\boldsymbol{\alpha}}$, if the channel is time invariant, $\boldsymbol{H}_{\boldsymbol{n}}$ is a convolutional matrix and the band structure of $\boldsymbol{H}_{\boldsymbol{d} \boldsymbol{f}}$, therefore, will be diagonal. However, the band structure of $\boldsymbol{H}_{\boldsymbol{d} f}$ over doubly selective channel is not diagonal, and energy spreads into adjacent subcarriers, contributing to ICI. The structure of $\boldsymbol{H}_{\boldsymbol{d} \boldsymbol{f}}$ becomes banded, with nonzero off-diagonal coefficients, most of which concentrates around the main diagonal. Notice that, the complexity grows linearly with the length of the OFDM block, thus introducing the requirement of low-complexity equalization. The binary matrix not only mitigates the adjacent channel interference, but also eliminates coupling components at the upper right and bottom left corner of $\boldsymbol{H}_{\boldsymbol{d} \boldsymbol{f}}$.

Due to the fact that the majority of components are distributed around the main diagonal, masked channel matrix is applied to reduce the computational complexity of $\mathbf{H}_{\mathbf{d f}}$, 
defined as

$$
\boldsymbol{B}= \begin{cases}{\left[\boldsymbol{H}_{\boldsymbol{f m}}\right]_{i,<i+u>_{N}+1}, \quad-U \leq u \leq U} \\ 0, \quad \text { ohterwise }\end{cases}
$$

where $\mathrm{U}$ could is selected to be proportional to the Doppler bandwidth, confining the non-zero elements to be confined to $\mathrm{U}$ off-diagonals above and below the main diagonal of $\boldsymbol{H}_{\boldsymbol{f m}}$.

\section{Selection of optimal order}

As mentioned above, the off-diagonal coefficients representing ICI spread around the main diagonal of $\boldsymbol{H}_{\boldsymbol{d} f}$, and the allocation of its power changes according to fractional order $\alpha$ of DFrCT-OFDM. When the system reaches its optimal order, the coefficients on or along the main diagonal will be largest, which means non-diagonal elements contributes to the least ICI. Meanwhile, it means that the sub channel carrier frequencies variation match the high timefrequency distortion of the channel. In this paper, the search of optimal order $\boldsymbol{\alpha}_{\text {opt }}$ is based on exploiting carrier-tointerference ratio (CIR), defined as follows

$$
C I R=\frac{\sum_{n=1}^{N}\|B(n, n)\|^{2}}{\sum_{k=1 k \neq n}^{N}\|B(n, k)-B(n, n)\|^{2}}
$$

where $\boldsymbol{H}_{\boldsymbol{d} \boldsymbol{f}}$ is replaced by the masked channel matrix $\boldsymbol{B}_{\boldsymbol{k}}$, as mentioned in 2.3. Subsequently, the optimal order can be estimated as a maximum $\boldsymbol{C I R}$ problem, as follows

$$
\alpha_{\text {opt }}=\arg \{\underset{\alpha \in[-1,1]}{\min }[\boldsymbol{C I R}]\}
$$

The iterative search approach can be applied within the range of $\alpha$ from-1to 1 .It is obvious that the selection of optimal order $\alpha$ depends on the channel properties, including number of subcarriers N, Doppler shift, number of resolvable paths and channel power delay profile. The optimal order $\boldsymbol{\alpha}_{\mathbf{o p t}}$ searched at the receiver is then sent back to the transmitter as illustrated in Fig 1.

\section{E. Tikhonov regularization based turbo MMSE equalization}

Turbo equalization is a Bayesian iterative method that is obtained by estimating prior information, including the mean

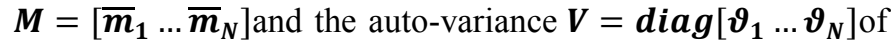
the symbols. Initially, there is no prior information, so we assume for all the symbols, the linear MMSE estimate of the $\mathbf{n}^{\text {th }}$ subcarrier is denoted as

$$
\hat{\boldsymbol{s}}_{\boldsymbol{n}}=\overline{\boldsymbol{m}}_{\boldsymbol{n}}+\boldsymbol{G}_{\boldsymbol{n}}{ }^{\boldsymbol{H}}\left(\boldsymbol{R}_{\boldsymbol{d f}}-\boldsymbol{B M}\right)
$$

Where the coefficients of $\mathbf{n}^{\text {th }}$ subcarrier is denoted as

$$
\boldsymbol{g}_{n}=\left(B V B^{H}+\gamma I\right)^{-1} b_{k} \boldsymbol{\vartheta}_{n}
$$

where $\gamma$ denotes the inverse of signal to noise ratio $\left(\mathrm{SNR}^{-1}\right)$.

However, the doubly selective channels could be highly ill-conditioned, which means that the ratio between the largest eigenvalue and the smallest eigen value of the channel matrix become very large. Therefore, at high SNR, the inverse matrix contained in the MMSE formula is subject to significant numerical errors, contributing to less stable of equalization. In order to solve this problem, a Tikhonov regularization based MMSE algorithm is proposed to replace the inverse of Signal to noise $\left(\mathrm{SNR}^{-1}\right)$ ratio by inversed modified signal to interference (SINR) ratio [12], which is described as follows

$$
S I N R=\frac{1-P o}{P o+\gamma}
$$

Where Po represents the out of band power, defined as

$$
\boldsymbol{P o}=\frac{\left\|H_{d f}-B_{k}\right\|^{2}}{N_{a}}
$$

Where $\mathbf{N}_{\boldsymbol{a}}$ represents the uncompensated ICI that falls in a specific data subcarrier.The total uncompensated ICI must be divided by the number $\mathbf{N}_{\boldsymbol{a}}$ of data subcarriers. Equation (10) therefore, can be changed to

$$
\boldsymbol{G}_{n}=\left(B V B^{H}+S I N R * I\right)^{-1} b_{k} \vartheta_{n}
$$

In addition, the matrix inversion of a banded matrix in the MMSE equalizer leads to a significant increase in computational complexity. $\boldsymbol{L D} \boldsymbol{L}^{\boldsymbol{H}}$ factorization equalization [3] is applied to exploit the band structure of $\boldsymbol{G}_{\boldsymbol{n}}$.

Based on the output of equalizer $\hat{\boldsymbol{s}}_{\boldsymbol{n}}$, the extrinsic loglikelihood ratio (LLR)is $\Delta \boldsymbol{L}\left(\hat{\boldsymbol{s}}_{\boldsymbol{n}}\right)$, which represents the soft information based on the maximum a posteriori (MAP) criterion.Assuming that the estimate $\hat{\mathbf{s}}_{\mathbf{n}}$ meets the requirement of conditionally Gaussian distribution with probability density function (PDF), then $\Delta \mathbf{L}\left(\hat{\mathbf{s}}_{\mathbf{n}}\right)$ may be written as

$$
\Delta L\left(\hat{s}_{n}\right)=p\left(\hat{s}_{n} \mid s_{n}\right)=\frac{1}{\pi \sigma_{n}^{2}} \exp \left(\frac{\left|\hat{s}_{n}-\mu_{n}\right|^{2}}{-\left(\sigma_{n}\right)^{2}}\right)
$$

Where $\widehat{\mu}_{n}$ and $\boldsymbol{\sigma}_{n}^{2}$ represent the mean and variance, which could be denoted as

$$
\begin{gathered}
\boldsymbol{\mu}_{n}=\boldsymbol{E}\left\{\hat{\boldsymbol{s}}_{\boldsymbol{n}} \mid \boldsymbol{s}_{n}\right\}=\overline{\boldsymbol{m}}_{\boldsymbol{n}}+\boldsymbol{G}_{\boldsymbol{n}}^{\boldsymbol{H}} \boldsymbol{b}_{\boldsymbol{k}}\left(\boldsymbol{s}_{\boldsymbol{n}}-\overline{\boldsymbol{m}}_{\boldsymbol{n}}\right), \quad \text { with } \mathbf{s}_{\mathrm{n}}= \pm \mathbf{1} \\
\boldsymbol{\sigma}_{n}^{2}=\operatorname{Cov}\left(\hat{\boldsymbol{s}}_{n}, \hat{\boldsymbol{s}}_{n} \mid \boldsymbol{s}_{n}\right)
\end{gathered}
$$

The aposteriori $\operatorname{LLRL}\left(\mathbf{s}_{\mathbf{n}} \mid \hat{\mathbf{s}}_{\mathbf{n}}\right)$ can be obtained by the sum of the extrinsic LLR $\Delta \mathbf{L}\left(\hat{\mathbf{s}}_{\mathbf{n}}\right)$ and the a priori $\operatorname{LLR} \mathbf{L}\left(\mathbf{s}_{\mathbf{n}}\right)$ as follows [7]:

$$
\frac{P\left(s_{n}=+1 \mid \hat{s}_{n}\right)}{P\left(s_{n}=-1 \mid \hat{s}_{n}\right)}=\ln \frac{p\left(s_{n} \mid s_{n}=+1\right)}{\underbrace{p\left(s_{n} \mid s_{n}=-1\right)}_{L\left(s_{n} \mid \hat{s}_{n}\right)}}+\underbrace{\frac{P\left(s_{n}=+1\right)}{P\left(s_{n}=-1\right)}}_{\Delta L\left(\hat{s}_{n}\right)}
$$

From the input data it is noted that the a priori LLR is available before the equalization. The calculation of the updated mean and auto-variance of the estimated symbol is as follows [8]:

$$
\begin{gathered}
\overline{\boldsymbol{m}}_{\text {n.new }}=\tanh \left(L\left(s_{n} \mid \hat{s}_{n}\right) / 2\right) \\
\vartheta_{n, \text { new }}=1-\left(\overline{\boldsymbol{m}}_{\text {n.new }}\right)^{2}
\end{gathered}
$$

After that, the updated means $\overline{\mathbf{m}}_{\text {n.new }}$ and variance $\boldsymbol{\vartheta}_{n, n e w}$ of current iteration is feedback to equalization, ready for next iteration. The posteriori LLR of current iteration $\boldsymbol{L}\left(\boldsymbol{s}_{\boldsymbol{n}} \mid \hat{\boldsymbol{s}}_{\boldsymbol{n}}\right)$ could be a priori LLR for the next iteration $\boldsymbol{L}_{\text {new }}\left(\boldsymbol{s}_{\boldsymbol{n}}\right)$.In our paper, the extrinsic soft information LLR $\Delta \boldsymbol{L}\left(\hat{\boldsymbol{s}}_{\boldsymbol{n}}\right)$ calculated from equalization is sent to convolutional decoder for further update of LLR information mean and auto-variance.

\section{Simulation result and discussion}

The DFrCT-Turbo is simulated, with its performance compared to that from the turbo equalization based on conventional OFDM and DFrCT-OFDM. The channel in this simulation is selected as doubly selective Rayleigh fading channel, with perfect channel state information. In addition, a rate $1 / 2$ convolution coding is incorporated to improve the performance. 


\section{A. $\quad$ Simulation set up}

The number of subcarriers is $\mathrm{N}=128$, of which 96 are active and the length of cyclic prefix is $\mathrm{L}=8$. The normalized Doppler frequency is $f_{d}=0.30 \Delta f$, where $\Delta f$ is the normalized subcarrier spacing. The UWA channel is modeled as Rayleigh fading channel with exponential multipath intensity profile of $[-7.2,-4.2,-6.2,-10.5,-12.2,-14.0] \mathrm{dB}$ and time delay profile of $[0,0.02,0.05,0.16,0.23,0.5] \mathrm{ms}$. The signal to noise ratio (SNR) ranges from 0 to $80 \mathrm{~dB}$. In addition, the low complexity equalizer is set at $\mathrm{U}=6$ and the number of Monte Carlo runs is 10000. [13]

\section{B. Simulation performance analysis}

Figure 2 compares the BER performance of the DFrCTTurbo, conventional OFDM scheme with Tikhonov regularizationbased turbo MMSE equalization and DFrCTOFDM. The optimal order obtained is 0.95 . It can be seen that performance of DFrCT-Turbo is superior to that of conventional OFDM with a BER improvement of $1 \mathrm{~dB}$. The reason of positive results is that non-zero band of the channel matrix in the fractional domain is lower than the conventional frequency domain, especially in doubly selective channel. In the other word,the allocation of the band of conventional OFDM distributes over more frequencies is than that of DFrCT, which means the energy of $\mathrm{H}_{\mathrm{df}}$ based on DFrCT concentrates closer to the diagonal, contributing to less ICI and better BER performance.

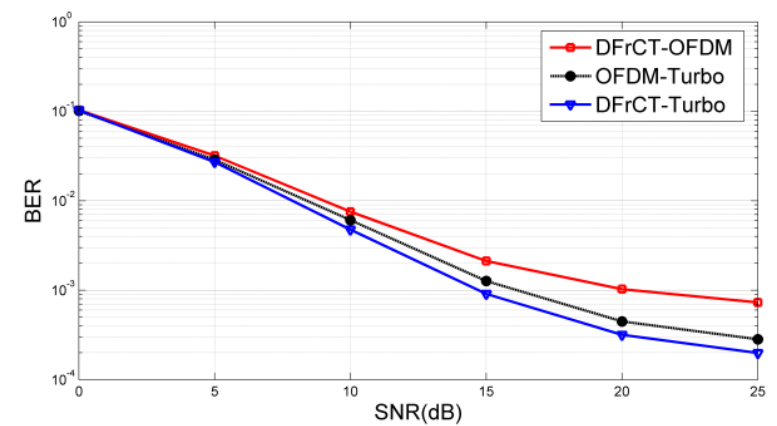

Figure2: BER of DFrCT-Turbo and OFDM-Turbo, and DFrCT-OFDM

Figure 2 shows that the DFrCT-Turbo is superior to DFrCT-OFDM by approximately up to $5 \mathrm{~dB}$, attributed to the iterative performance of Tikhonov regularization based turbo MMSE equalization

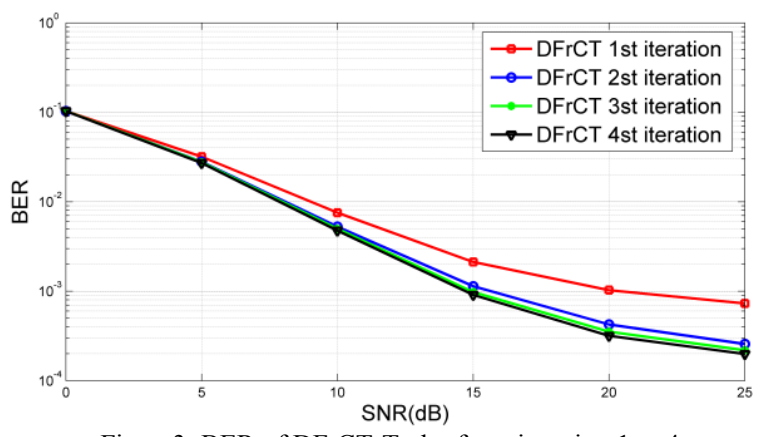

Figure3: BER of DFrCT-Turbo from iteration 1 to 4

Figure 3 shows the performance of the proposed DFrCT-
Turbo at different equalization iterations. It can be seen that the BER improves as the number of iteration increases converging to the optimum at the $4^{\text {th }}$ iteration.

Figure 4 compares the performance of the proposed turbo equalization of DFrCT-OFDM turbo with or without error correction coding, which is a rate $1 / 2$ convolutional code with generator matrix [ $\left.\begin{array}{llllll}1 & 0 & 1,1 & 1 & 1\end{array}\right]$ and random interleaving. The iteration number is set at 2 . It is obvious that the system with ECC code outperforms with significant BER improvement of $30 \mathrm{~dB}$.

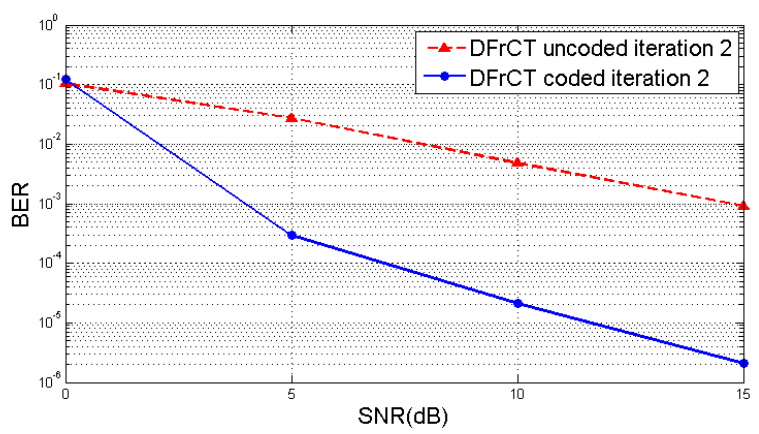

Figure4: Comparison of BER of DFrCT with or without convolutional coding

\section{Conclusion}

A novel DFrCT-Turbo system based on the hybrid use of the Discrete Fractional Cosine Transform (DFrCT), Tikhonov regularization based turbo MMSE equalization and low complexity banded MMSE equalization, and has been presented. The simulation results demonstrate that ICI is significantly mitigated under doubly selective channel compared to the conventional OFDM at moderate complexity providing an improvement in the overall Bit Error Rate.

\section{ACKNOWLEDGEMENT}

This work was supported by the Engineering and Physical Sciences Research Council (EPSRC) Grant number EP/K014307/1 and the MOD University Defence Research Collaboration in Signal Processing.

\section{REFERENCE}

[1] M. Stojanovic and J. Preisig, "Underwater acoustic communication channels: Propagation models and statistical characterization," IEEE Commun. Mag, vol. 47, no. 1, pp. 84-89, Jan. 2009.

[2] Y M. Aval, and M. Stojanovic, "Differentially Coherent Multichannel Detection of Acoustic OFDM Signals," IEEE Journal of Oceanic Engineering.

[3] L. Rugini, P. Banelli, and G. Leus, "Low-complexity banded equalizers for OFDM systems in Doppler spread channels," EURASIP Journal on Applied Signal Processing, vol. pp. 1-13, 2006.

[4] B. Li, S. Zhou. M. Stojanovic, L. Freitag, and P. Willet, "Multicarrier Communication over underwater acoustic channels with non-uniform Doppler shifts," IEEE J. Ocean. Eng., vol. 33, no. 2, pp. 198-209, Apr. 2008.

[5] J. Huang, S. Zhou, J. Huang, C.R. Berger, and P. Willett, "Progressive inter-carrier interference equalization for OFDM 
transmission over time-varying underwater acoustic channels, "IEEE J. Sel. Topics in signal process., vol. 5, no. 8, pp. 1524-1536, 2011.

[6] K. Fang, L. Rugini, and G. Leus, "Low-complexity block turbo equalization for OFDM systems in time-varying channels," IEEE Trans. Signal Process., vol.56, no. 11, pp. 5555-5566, Nov. 2008.

[7] P. Schniter, "Low-complexity equalization of OFDM in doubly selective channels," IEEE Transactions on Signal Processing, vol. 52, no. 4, pp. 1002-1011, April 2004.

[8] S. Ahmed, M. Sellathurai, S. Lambotharan, and J. A. Chambers, "Low complexity iterative method of equalization for single carrier with cyclic prefix in doubly selective channels," IEEE Signal Process. Letter,. Vol. 13, no. 1, pp. 5-8, Jan. 2006.

[9] A. Solyman, S. Weiss and J.J. Soraghan, "Hybrid DFrFT and FFT based Multimode Transmission OFDM System." presented at the ICEENG, 2012 Cairo, Egypt.
[10] A. Solyman, S. Weiss and J.J. Soraghan, "A Novel Orthogonal Chirp Division Multiplexing (OCDM) Multicarrier Transceiver Based on the Discrete Fractional Cosine Transform." Presented at the MIC-WCMC, 2013 Valencia, Spain, 2013

[11] P. Soo-Chang and D. Jian-Jiun, "Fractional cosine, sine, and Hartley transforms," Signal Processing, IEEE Transactions on, vol. 50, pp. 1661-1680, 2002.

[12] L. Rugini, P. Banelli, and G. Leus, "OFDM communications over time-varying channels", Chapter7 of Wireless Communications over Rapidly Time-Varying Channels, eds. F. Hlawatsch and G. Matz, Academic Press, 2011.

[13] A. Solyman, S. Weiss and J.J. Soraghan, "Low-Complexity LSMR Equalization of FrFT-Based Multicarrier Systems in Doubly Dispersive Channels, " presented at the ISSPIT 2011, Bilbao Spain, 2011. 